cause for concern. Reviewing the service after the nurses have more experience running it and estimating the real cost effectiveness outside the artificial restrictions of a trial would be useful. It would also be interesting to study the longer term effects of the nurses' service on patients' attitudes to their illnesses and behaviour in seeking health care.

Various members of the South Thames Research Network provided invaluable support during all stages of this study, in particular Dr Sarah Clement. The network is funded by the South East and London regions of the NHS Executive. Statistical analysis was supervised by Dr Pak Sham. Thanks are also owed to the staff of the five practices, to the nurses who volunteered to participate in the study, and to the patients who took part.

Contributors: CS initiated and coordinated the formulation of the hypothesis, discussed core ideas, designed the study protocol and questionnaires, analysed the data, acted as overall coordinator for the trial, and participated in writing the paper CS is guarantor for the paper. AH helped formulate the core ideas and the study protocol, participated in data collection, and contributed to writing the paper. DW helped formulate the core ideas and study protocol and participated in writing the paper. MAC helped formulate the hypothesis and protocol and participated in writing the paper. SK participated in collecting the data and coordinating the day to day running of the study. SC helped formulate the hypothesis and core ideas, designed the protocol for data analysis, and participated in writing the paper.

Funding: This project was funded by the project grant scheme of the South Thames region of the NHS Executive. Competing interests: None declared.

1 Secretary of State for Health. The new NHS. London: Stationery Office, 1997. (Cm 3807.)

2 Marsh G, Dawes M. Establishing a minor illness nurse in a busy general practice. BMJ 1995;310:778-80.

Rees M, Kinnersley P. Nurse-led management of minor illness in a GP surgery. Nurs Times 1992;6:32-3.

4 Baker R. Consultation satisfaction questionnaire: development of a questionnaire to assess patients' satisfaction with consultations in general practice. BrJ Gen Pract 1990;40:487-90.

5 Poulton B. Use of the consultation satisfaction questionnaire to examine patients' satisfaction with general practitioners and community nurses: reliability, replicability, and discriminant validity. Br J Gen Pract 1996;46: 26-31.

6 Murphy AW, Bury G, Plunkett PK, Gibney D, Smith M, Mullan E Randomised controlled trial of general practitioner versus usual medical care in an urban accident and emergency department: process, outcome, and comparative cost. BMJ 1996;312:1135-41.

7 Campbell A, Kearsley N, Herdman M, Maric S. Establishing a mino illness nurse in a busy general practice: may reduce doctors' workload. BMJ 1995;310:1404-5.

(Accepted 15 March 2000)

\author{
Abstract \\ Objective To ascertain any differences between care \\ from nurse practitioners and that from general \\ practitioners for patients seeking "same day" \\ consultations in primary care. \\ Design Randomised controlled trial with patients \\ allocated by one of two randomisation schemes (by \\ day or within day). \\ Setting 10 general practices in south Wales and south \\ west England. \\ Subjects 1368 patients requesting same day \\ consultations. \\ Main outcome measures Patient satisfaction, \\ resolution of symptoms and concerns, care provided \\ (prescriptions, investigations, referrals, recall, and \\ length of consultation), information provided to \\ patients, and patients' intentions for seeking care in \\ the future. \\ Results Generally patients consulting nurse \\ practitioners were significantly more satisfied with \\ their care, although for adults this difference was not \\ observed in all practices. For children, the mean \\ difference between general and nurse practitioner in \\ percentage satisfaction score was $-4.8(95 \%$ \\ confidence interval -6.8 to -2.8 ), and for adults the \\ differences ranged from $-8.8(-13.6$ to -3.9$)$ to 3.8 \\ ( -3.3 to 10.8$)$ across the practices. Resolution of \\ symptoms and concerns did not differ between the
}

two groups (odds ratio 1.2 (95\% confidence interval 0.8 to 1.8 ) for symptoms and 1.03 (0.8 to 1.4 ) for concerns). The number of prescriptions issued, investigations ordered, referrals to secondary care, and reattendances were similar between the two groups. However, patients managed by nurse practitioners reported receiving significantly more information about their illnesses and, in all but one practice, their consultations were significantly longer. Conclusion This study supports the wider acceptance of the role of nurse practitioners in providing care to patients requesting same day consultations.

\section{Introduction}

General practices need to provide care for patients who request "same day" consultations because they are too ill or otherwise unable to wait for an appointment. The numbers of these "extra" patients are difficult to predict and increasing. ${ }^{1}$ They are normally seen by general practitioners, although recently nurse practitioners have taken on this work. ${ }^{2-4}$ The Royal College of Nursing has developed training for nurse practitioners, although there is no requirement for nurses seeing these patients to hold specific qualifications.

Previous studies of nurse practitioners have found high levels of patient satisfaction, low levels of prescribing, and little need to refer patients to general practitioners. ${ }^{45}$ However, these studies were observa-
Department of General Practice, University of Wales College of Medicine,

Llanedeyrn Health

Centre, Cardiff CF3 7PN

Paul Kinnersley senior lecturer Elizabeth Anderson research officer Chris C Butler senior research fellow continued over

BMJ 2000;320:1043-8

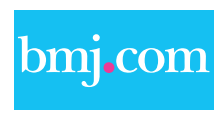

Two further tables of results are available on the BMJ's website 
Research and Development Support Unit, Trust Headquarters,

Southmead

Hospital, Bristol

BS10 5NB

Kate Parry

statistician

Andrew

Stainthorpe

research and

development

coordinator

Chris Rogers

senior statistician

Gloucester Road Medical Centre, Horfield, Bristol BS7 8SA

John Clement general practitioner Aileen Fraser nurse practitioner

London School of Economics Health, London

WC2A 2AE

Luke Archard research officer

Bristol Cancer Help Centre, Bristol BS8 4PG

Pat Turton director of education

Correspondence to: Paul Kinnersley kinnersley@cf.ac.uk tional and usually involved single practitioners. Our aim was to investigate whether nurse practitioner care differs from general practitioner care for patients requesting same day consultations.

\section{Methods}

\section{Recruitment of clinicians}

Nurse practitioners were defined as nurses employed in general practice who had completed the nurse practitioner diploma course at either the Royal College of Nursing Institute of Advanced Nursing, or the department of nursing, midwifery, and health care, University of Wales. All nurse practitioners who had completed this training at least one year previously and were working in south Wales or south west England were contacted by their educational institutions. Practices that expressed interest were visited. Relevant local research ethics committees approved the study.

\section{Recruitment of patients and randomisation}

Patients seeking a 'same day' consultation were recruited. Originally we planned to randomise patients to general practitioner or nurse practitioner care by day of consulting. However, this strategy was not acceptable to all practices so we offered two methods of randomisation (by day and within day). and allowed practices to choose their preferred method.

Patients requesting same day appointments who were prepared to consult either a general practitioner or a nurse practitioner were informed about the study in general terms. Consent was obtained when patients attended the surgery, and they were told which clinician they would see. All practices had a trained member of staff (the project coordinator) to manage the study procedure. under the supervision of the project research officer. The randomisation schemes were generated at the department of general practice in Cardiff, University of Wales College of Medicine.

In practices using randomisation by day, all patients consulting on a particular day saw the same type of practitioner. Practices were supplied with a calendar of their study period with the days allocated at random as nurse practitioner or general practitioner days by block randomisation. Block randomisation was used to ensure balance between the days allocated to the two types of practitioner.

Some of the practices that chose to randomise patients within day had appointments for same day patients fitted in throughout the day while others had unbooked consulting sessions. For practices which had fitted in appointments, the order in which the appointments were to be used was organised according to the block randomisation scheme provided. Sequential, consenting patients were allocated to the consultation slots when they contacted the practices. In the practices that allocated unbooked sessions, patients were allocated on arrival by block randomisation used to ensure a balanced allocation of patients on each day. Patients who seemed too ill or unable to understand the research and women seeking emergency contraceptive advice were excluded. The latter group was excluded to avoid embarrassment to those who might not wish to receive a postal questionnaire. General practitioners were always available to prescribe when necessary.
Outcome measurement and data collection

The primary outcomes were patient satisfaction immediately after the consultation, resolution of symptoms at two weeks, and resolution of concerns at two weeks. ${ }^{6}$ Secondary outcomes included care in the consultation (length of consultation, information provided), resource use (prescriptions, investigations, referrals), follow up consultations, and patients' intentions for dealing with future similar illnesses.

Two patient questionnaires were developed. The first (exit questionnaire) was administered at the time of the consultation. Before the consultation, patients recorded their levels of discomfort and concern on Likert-type scales and provided demographic details. After the consultation, they completed the consultation satisfaction questionnaire ${ }^{8}$ and answered yes or no to questions on the information provided by the clinician during the consultation (the cause of the illness, what the patient could do to relieve symptoms, likely duration, how to reduce chances of recurrence, and what the patient should do if the problem didn't improve). Completed questionnaires were returned to the project coordinator.

The consultation satisfaction questionnaire has been used by adults to rate general practitioners and nurse practitioners ${ }^{89}$ but not by parents consulting about children. After discussion with the originator of the instrument, we modified the items and tested this questionnaire against the paediatric medical interview satisfaction scale ${ }^{11}$ with 62 patients in a Cardiff practice. The mean difference between scores was -0.33 (SD 7.18) and the limits of agreement were -15.28 to $12.62,{ }^{12}$ suggesting no systematic bias between the two methods. The non-completion rate on the paediatric medical interview satisfaction scale was higher than for the modified consultation satisfaction questionnaire. We concluded that the modified consultation satisfaction questionnaire was a reasonable measure of satisfaction for children's consultations and used it for all patients aged 15 or younger.

A second questionnaire was sent to all patients two weeks after their consultation. Patients were asked to record resolution of symptoms and their current level of concern on Likert-type scales, whether they had sought further advice, and how they would deal with future similar illnesses. A single reminder was sent to non-respondents.

Clinicians completed an encounter sheet for each patient, recording length of consultation (including, for the nurse practitioners, any breaks taken); the patient's presenting illness; prescriptions issued; investigations ordered; referrals to other clinicians; and if the patient was asked to reattend.

Four weeks after the initial consultations, patients' medical records were checked for reattendance or hospital admission for the same problem. The results were recorded on an 'audit sheet'.

\section{Statistical methods}

Responses to items on the consultation satisfaction questionnaire were scored on a 1-5 scale, where $5=$ very satisfied and $1=$ very dissatisfied. Items scores were summed to produce a total unless data for any component question were missing. Total scores were converted to percentages for analysis. Patient satisfaction was analysed separately for adult and child 
consultations as the modified consultation satisfaction questionnaire used for children contained one fewer question.

We coded social class from the patient's stated occupation using the Office of Population and Censuses Surveys 1991 standard occupation classification. ${ }^{13}$ Information on morbidity was obtained from the clinician encounter sheet and the medical records during the audit and this information was categorised to produce a final morbidity coding scheme based on the Royal College of General Practitioners and Office of Population and Censuses Surveys coding system. ${ }^{14}$

\section{Sample size calculation}

Previous studies found mean satisfaction scores of $76.7 \%$ (SD 11.4) and that $65 \%$ of patients reported resolution of symptoms at two weeks. ${ }^{75}$ Taking a 5\% difference in satisfaction and a $10 \%$ difference for resolution of symptoms as being of clinical importance, we calculated that sample sizes of 220 and 900 patients were needed for the two outcomes to give $90 \%$ power at a significance level of 5\%. An inflation factor of 1.5 was used to account for the clusters of patients randomised by day, and we expected to achieve a $70 \%$ response rate to the postal questionnaire, giving a recruitment target of 2000 patients to examine both outcomes.

\section{Analyses}

Since we used both simple randomisation (within day) and cluster randomisation (by day), we had to assess the effect of the cluster randomisation. We calculated intraclass correlations for each outcome for the practices that used cluster (by day) randomisation using the proc mixed procedure and glimmix macro within SAS software.

All analyses reported include an adjustment for general practice. A general linear model, assuming normally distributed errors, was fitted to the consultation satisfaction questionnaire data and to the consultation time data. Log consultation times were analysed to minimise departures from the model assumptions. Logistic regression was used to compare the two groups for binary outcomes. For these analyses, resolution of symptoms and concerns were grouped into improved (yes or no) and concerned (yes or no) respectively. The results are presented as treatment differences and 95\% confidence intervals. A $5 \%$ significance level was used throughout.

\section{Results}

\section{Recruitment of practitioners}

Twenty seven nurse practitioners were identified and sent information. Eighteen expressed interest, seven did not respond, and two declined. Of the 18, 12 were visited and six declined to take part after receiving further information. Ten practices finally agreed to participate (five in south Wales and five near Bristol), with list sizes ranging from 6000 to 16300 patients. One nurse worked in two practices, both of which took part, and one practice had two nurse practitioners. All were regularly seeing patients requesting same day appointments. No information was gathered on practices that declined to take part.

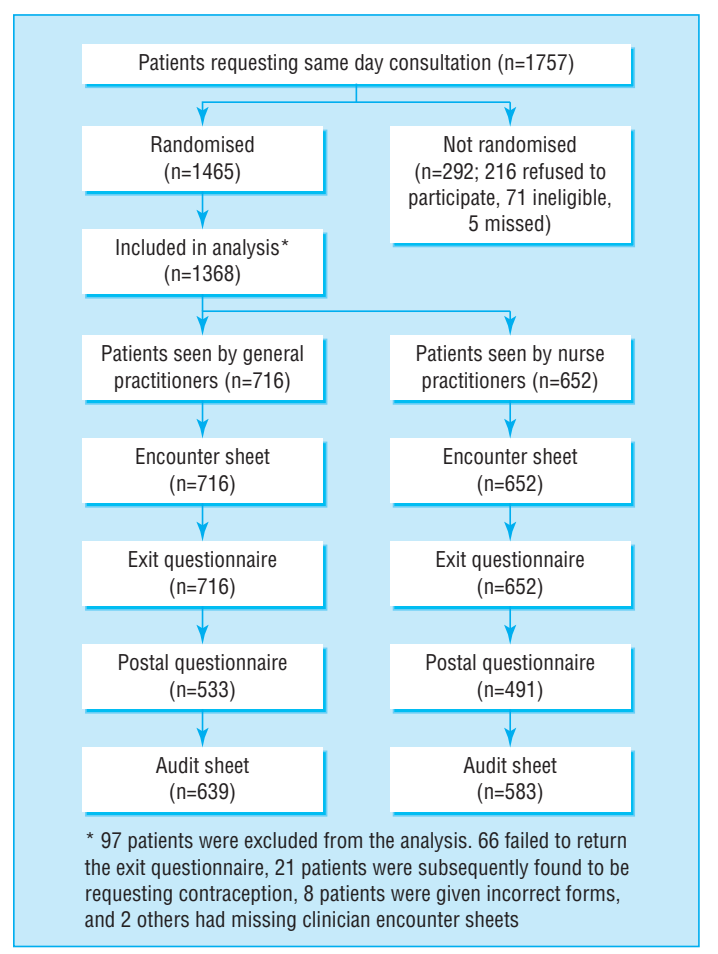

Flow chart showing patient recruitment and follow up

\section{Randomisation and intraclass correlations}

Six practices chose within day randomisation and four chose cluster randomisation by day. The intraclass correlations could not be estimated for three secondary outcomes. Of the 14 intraclass correlations that could be estimated, nine were less than 0.05 and five were between 0.05 and 0.13 . These were considered sufficiently small to assume statistical independence within a cluster. We therefore combined data from the two randomisation schemes and conducted analyses at the individual level.

\section{Patient recruitment}

The figure shows the flow of patients through the study; 1757 patients requested same day consultations, and data for 1368 were analysed. The patients in the two groups were similar in terms of age, sex, and social class (table 1). In all, 1024 patients $(75 \%)$ completed the postal questionnaire at two weeks. Audit data from the medical records were available for 1222 patients (89\%).

There were no notable differences between the two groups in terms of morbidity (table 2) or in initial degree of discomfort or concern. The commonest illnesses presented were respiratory diseases. Eighty nine per cent of patients (632) consulting a general practitioner and 90\% (576) of patients consulting a nurse practitioner reported some or a great deal of discomfort. Sixty six per cent of patients (465) consulting a general practitioner and 65\% (418) of patients consulting a nurse practitioner reported they were fairly or very concerned.

\section{Resolution of symptoms and concerns and patient satisfaction}

At two weeks, most patients reported that their symptoms had improved and their concerns were reduced. There were no notable or significant 
Table 1 Age, sex, and socioeconomic characteristics of patients studied

\begin{tabular}{lcc} 
& \multicolumn{2}{c}{ No (\%) seeing: } \\
\cline { 2 - 3 } & $\begin{array}{c}\text { General practitioner } \\
(\mathbf{n}=716)\end{array}$ & $\begin{array}{c}\text { Nurse practitioner } \\
(\mathbf{n}=652)\end{array}$ \\
\hline Age & $228(32)$ & $244(38)$ \\
\hline $0-15$ & $211(30)$ & $184(28)$ \\
\hline $16-35$ & $181(25)$ & $145(22)$ \\
\hline $36-55$ & $76(11)$ & $63(10)$ \\
\hline $56-75$ & $15(2)$ & $12(2)$ \\
\hline$>75$ & $711(100)$ & $648(100)$ \\
\hline Total & & \\
\hline Sex & $275(42)$ & $238(39)$ \\
\hline Male & $384(58)$ & $373(61)$ \\
\hline Female & $659(100)$ & $611(100)$ \\
\hline Total & & \\
\hline Social class & $40(7)$ & $39(7)$ \\
\hline I & $218(36)$ & $173(32)$ \\
\hline II & $161(26)$ & $137(25)$ \\
\hline III non- manual & $95(16)$ & $102(19)$ \\
\hline III manual & $71(12)$ & $65(12)$ \\
\hline IV & $25(4)$ & $24(4)$ \\
\hline V & $610(100)$ & $540(100)$ \\
\hline Total & &
\end{tabular}

Table 2 Presenting illnesses of patients

\begin{tabular}{lcc} 
Category of disease & $\begin{array}{c}\text { No }(\%) \\
\text { seeing general } \\
\text { practitioner } \\
(\mathbf{n = 6 9 2 )}\end{array}$ & $\begin{array}{c}\text { No (\%) } \\
\text { seeing nurse } \\
\text { practitioner } \\
(\mathbf{n = 6 2 6})\end{array}$ \\
\hline Respiratory system & $202(29)$ & $181(29)$ \\
\hline Nervous system and sensory organs & $101(15)$ & $90(14)$ \\
\hline Skin & $80(12)$ & $69(11)$ \\
\hline Musculoskeletal system & $60(9)$ & $46(7)$ \\
\hline Digestive system & $59(9)$ & $47(8)$ \\
\hline Allergic, endocrine, nutritional, and metabolic & $39(6)$ & $48(8)$ \\
\hline Genitourinary system & $37(5)$ & $32(5)$ \\
\hline Miscellaneous & $114(16)$ & $113(18)$ \\
\hline
\end{tabular}

differences between the two modes of care (table 3). The distribution of satisfaction scores was negatively skewed for general practitioner consultations, but for nurse practitioner consultations the scores followed a normal distribution. For children, satisfaction levels were significantly higher for nurse practitioner consultations compared with general practitioner consultations (table 3). There was a significant interaction between mode of care and practice for adults. Significantly higher satisfaction levels for nurse practitioner consultations were observed in three practices, but no significant differences were found in the remaining seven (table 4 ).

\section{Care provided}

There were no notable differences between the groups in terms of prescriptions issued, investigations ordered, or referrals to secondary care (table 5). Further details for outcomes where odds ratios varied significantly between practices are available on the $B M J$ 's website. At three of the 10 practices significantly more patients who saw a nurse practitioner were asked to reattend. However, the percentages of patients who actually reconsulted were similar.

In all but one practice, nurse practitioner consultations were significantly longer than general practitioner consultations. The ratio of consultation times between general and nurse practitioners ranged from
$0.46(95 \%$ confidence interval 0.39 to 0.54$)$ to 0.90 (0.70 to 1.13). In eight practices the nurse practitioner consultations were significantly longer even after breaks in the consultation (to get prescriptions signed or for other reasons) were excluded. The ratio of consultation times ranged from 0.57 (0.49 to 0.67$)$ to 0.92 ( 0.70 to 1.21$)$ after breaks were excluded (see BMJ's website for further details).

Significantly more patients who consulted a nurse practitioner reported that they had been told the cause of their illness, how to relieve their symptoms, and what to do if the problem persisted (table 5). Also, more patients reported being told the likely duration of their illness and how they could reduce the chance of recurrence, although these differences were significant in only three practices.

Of the patients who consulted a general practitioner, $73 \%$ (364) stated that they would consult a

Table 3 Resolution of symptoms and concerns at two weeks and patient satisfaction immediately after the consultation

\begin{tabular}{|c|c|c|}
\hline & $\begin{array}{c}\text { General } \\
\text { practitioner }\end{array}$ & $\begin{array}{c}\text { Nurse } \\
\text { practitioner }\end{array}$ \\
\hline \multicolumn{3}{|c|}{ Resolution of symptoms (№ (\%) of patients) ${ }^{*}$} \\
\hline Much better & $259(49)$ & $235(49)$ \\
\hline Better & $191(36)$ & $166(34)$ \\
\hline Unchanged & $65(12)$ & $71(15)$ \\
\hline Worse & $10(2)$ & $10(2)$ \\
\hline Much worse & $4(1)$ & $2(0.4)$ \\
\hline Total & $529(100)$ & $484(100)$ \\
\hline \multicolumn{3}{|c|}{ Resolution of concerns (No (\%) of patients) $\dagger$} \\
\hline Not concerned & $233(44)$ & $221(46)$ \\
\hline Little concerned & $187(35)$ & $173(36)$ \\
\hline Fairly concerned & $78(15)$ & $67(14)$ \\
\hline Very concerned & $31(6)$ & $23(5)$ \\
\hline Total & $529(100)$ & $484(100)$ \\
\hline \multicolumn{3}{|l|}{ Patient satisfaction } \\
\hline \multicolumn{3}{|l|}{ Adults: } \\
\hline No of patients & 403 & 334 \\
\hline Median (interquartile range) score & $74(67-80)$ & $77(70-82)$ \\
\hline \multicolumn{3}{|l|}{ Children: } \\
\hline No of patients & 193 & 210 \\
\hline Median (interquartile range) score & $76(69-82)$ & $79(73-87)$ \\
\hline Mean satisfaction scoreł & 75.62 & 80.40 \\
\hline Difference in mean score & \multicolumn{2}{|c|}{$-4.78(95 \% \mathrm{Cl}-6.75$ to -2.80$)$} \\
\hline
\end{tabular}

* Odds ratio (doctor/nurse) for symptom improvement $=1.23$ ( $95 \% \mathrm{Cl} 0.87$ to ${ }^{*}$ Odds

†Odds ratio (doctor/nurse) for not concerned adjusted for general practice $=1.03$ (95\% Cl 0.80-1.33).

fLeast squares means estimated from fitted model. No overall mean calculated for adults because of interaction between mode of care and practice (see table 4).

Table 4 Difference in mean percentage satisfaction score for adults by general practice

\begin{tabular}{lccc} 
& \multicolumn{2}{c}{ Mean satisfaction score } & \\
\cline { 2 - 3 } Practice & $\begin{array}{c}\text { General } \\
\text { practitioner }\end{array}$ & $\begin{array}{c}\text { Nurse } \\
\text { practitioner }\end{array}$ & Difference $(95 \%$ Cl) \\
\hline 1 & 68.86 & 77.65 & $-8.79(-13.59$ to -3.98$)$ \\
\hline 2 & 72.83 & 72.88 & $-0.05(-3.96$ to 3.87$)$ \\
\hline 3 & 79.47 & 75.72 & $3.75(-3.24$ to 10.74$)$ \\
\hline 4 & 71.45 & 75.41 & $-3.96(-7.70$ to -0.22 \\
\hline 5 & 68.66 & 74.58 & $-5.92(-15.70$ to 3.86$)$ \\
\hline 6 & 71.58 & 79.53 & $-7.95(-13.58$ to -2.31$)$ \\
\hline 7 & 75.02 & 74.41 & $0.61(-4.84$ to 6.05$)$ \\
\hline 8 & 74.28 & 77.49 & $-3.21(-8.71$ to 2.29$)$ \\
\hline 9 & 78.70 & 79.24 & $-0.54(-4.88$ to 3.81$)$ \\
\hline 10 & 70.93 & 76.83 & $-5.90(-12.11$ to 0.31$)$ \\
\hline
\end{tabular}

*Least squares means estimated from the fitted model. 
general practitioner for a similar illness in the future and only $8 \%$ (38) indicated that they would consult a nurse (table 5). Of those who saw a nurse practitioner, $48 \%$ (211) stated they would consult a general practitioner next time and 32\% (139) that they would consult a nurse. However, in six practices the number of patients who would consult a general practitioner in future was not significantly different between the two groups. In the remaining four practices, significantly more patients in the general practitioner group intended to seek general practitioner care in future.

\section{Discussion}

We found that patients who consulted nurse practitioners were generally more satisfied with their care, although the differences were less than the level of clinical importance used in the sample size calculation. The variation in mean satisfaction scores for adults between practices suggests that individual clinicians have a big influence. The nurse practitioner consultations were significantly longer and their patients reported being provided with more information. There were no notable differences for the other outcomes studied.

The imposition of the study procedure changed the working arrangements within the practices. We attempted to minimise this by providing flexibility over the method of randomisation. Practices that are considering introducing nurse practitioner care should offer patients a choice.

We are unaware of other studies comparing the information provided by doctors and nurses. Here most patients reported that their clinician provided information on what to do if symptoms persisted, although lower levels of provision were reported for other important information. ${ }^{16}$ The nurses' consultations may be longer because they provide more information or because of different time constraints. Longer consultations and those in which more information is provided have been previously associated with greater satisfaction. ${ }^{17} 18$

Our sample size was smaller than our target based on the assumption that all patients would be randomised by day. However, only four practices chose randomisation by day, and since we found that clustering could be ignored and the combined dataset analysed at the patient level, our sample size exceeded the estimated 900 needed.

Most patients reported high levels of discomfort and concern before their consultation. The questionnaires seem to be responsive since most patients reported reduced symptoms and concerns at two weeks. This may be due to effective treatments or the self limiting nature of the illnesses. If the illnesses were self limiting, it is unsurprising that we found no differences between the two groups in terms of resolution of symptoms.

Patients requiring same day appointments are a diverse group. A third of patients were either not concerned or a little concerned, raising the question of why they consulted. However, some patients may present with early symptoms of serious conditions. The detection of such cases would be important in judging the overall quality of care, but a different study design would be needed.
Table 5 Care provided within consultations and patient intentions for managing future illnesses. Values are numbers (percentages) of patients unless stated otherwise

\begin{tabular}{|c|c|c|c|}
\hline & $\begin{array}{c}\text { General } \\
\text { Practitioner } \\
(\mathrm{n}=716)\end{array}$ & $\begin{array}{c}\text { Nurse } \\
\text { Practitioner } \\
(n=652)\end{array}$ & Odds ratio $(95 \% \mathrm{Cl})^{*}$ \\
\hline \multicolumn{4}{|l|}{ Treatment action } \\
\hline Prescription issued & $434(63)$ & $407(63)$ & $1.01(0.80$ to 1.28$)$ \\
\hline Investigation ordered & $73(11)$ & $80(12)$ & $0.83(0.58$ to 1.16$)$ \\
\hline Referred & $34(5)$ & $33(5)$ & $0.96 \dagger(0.58$ to 1.57$)$ \\
\hline Follow up advised $\ddagger$ & $168(25)$ & $222(35)$ & $\begin{array}{l}0.11(0.03 \text { to } 0.37) \text { to } \\
1.41(0.71 \text { to } 2.80)\end{array}$ \\
\hline Reconsulted for same problem & $182(29)$ & 177 (31) & $0.91(0.70$ to 1.17$)$ \\
\hline \multicolumn{4}{|l|}{ Provision of information } \\
\hline Cause of illness & 491 (72) & $501(81)$ & $0.58(0.44$ to 0.76$)$ \\
\hline Relief of symptoms & 467 (68) & $548(86)$ & $0.32(0.24$ to 0.43$)$ \\
\hline Duration of illness & $388(57)$ & $404(64)$ & $\begin{array}{l}0.34 \text { (0.14 to } 0.84) \text { to } \\
2.38(0.79 \text { to } 7.14)\end{array}$ \\
\hline How to reduce chance of recurrence $\ddagger$ & $139(21)$ & $205(34)$ & $\begin{array}{l}0.19 \text { (0.09 to } 0.38) \text { to } \\
1.57(0.46 \text { to } 5.23)\end{array}$ \\
\hline What to do if problem persists & $604(88)$ & $584(93)$ & $0.61(0.41$ to 0.90$)$ \\
\hline \multicolumn{4}{|l|}{ Intentions for future treatment } \\
\hline Treat self & $48(10)$ & $50(11)$ & - \\
\hline Consult general practitionerł & $364(73)$ & $211(48)$ & $\begin{array}{l}0.76(0.10 \text { to } 5.48) \text { to } \\
11.94(2.11 \text { to } 67.3)\end{array}$ \\
\hline Consult nurse & $38(8)$ & $139(32)$ & - \\
\hline Other & $48(10)$ & $37(9)$ & - \\
\hline
\end{tabular}

Length of consultation ( $\mathrm{min}$ )

\begin{tabular}{lccc}
\hline No of patients & 648 & 639 & - \\
\hline Median (interquartile range) & $6(4-8)$ & $10(7-14)$ & - \\
\hline Median (interquartile range) excluding breaks & NA & $8(6-11)$ & -
\end{tabular}

Median (interquartile range) excluding breaks

${ }^{*}$ Adjusted for general practice.

†Because of small number of referrals it was not possible to adjust for general practice.

$\ddagger$ Range reported because odds ratios varied significantly across practices.

Previous observational studies found lower levels of prescribing by nurse practitioners and different patterns of patient morbidity. ${ }^{4}$ We did not find this. As they were given more information, patients seen by nurse practitioners might be expected to cope more effectively with similar illnesses in future. However, similar, small proportions of each group reported that they would self manage future illnesses. This may reflect the contrary effect of prescribing, which was similar in both groups and validates the patient's decision to seek help.

\section{What is already known on this topic}

General practices have to provide care to patients who request same day consultations

Nurse practitioners have extended their role to managing these patients

Care of these patients by nurse practitioners and general practitioners has not been compared in randomised trials

\section{What this study adds}

Patients who consulted nurse practitioners received longer consultations, were given more information, and were generally more satisfied

There were no differences for a range of other outcomes, including resolution of symptoms and concerns and prescribing

The study supports the extension of the role of nurse practitioners to include seeing patients requesting same day consultations 
The demands placed on practices mean that they may explore alternative methods of management for same day patients. However, the overall use of resources within the NHS must be considered before widespread changes are made. Nevertheless, the positive outcomes found here suggest that nurses provide a high standard of care to their patients, and this supports their extended role within primary care.

We thank the patients, nurses, and doctors who took part in this study. Professors Debbie Sharp, Nigel Stott and Richard Baker provided additional valuable support and advice.

Contributors: JC and AF initiated this study. PT, LA, and AS developed the methods. PK and EA further developed the methods and undertook data collection. CR and KP undertook the analysis. Eileen O'Donnell was responsible for data processing. All authors were involved in the interpretation of the results and writing the report. PK and CR act as guarantors for this study.

Funding: The research was supported by a grant from the Welsh Office of Research and Development for Health and Social Care. CB is supported by a fellowship from the Welsh Office of Research and Development for Health and Social Care. The Research and Development Support Unit at Southmead Hospital is supported by a grant from South West NHS Research and Development Directorate.

Competing interests: None declared.

1 Gill D, Dawes M, Sharpe M, Mayou R. GP frequent consulters: their prevalence, natural history, and contribution to rising workload. BrJ Gen Pract 1998;48:1856-7.

2 Marsh GN, Dawes ML. Establishing a minor illness nurse in a busy general practice. $B M J$ 1995;310:778-80.

3 Rees M, Kinnersley P. Nurse management of minor illness in general practice. Nursing Times 1996:92:32-3.

4 Myers P, Lenci B, Sheldon MG. A nurse practitioner as the first point of contact for urgent medical problems in a general practice setting. Fam Pract 1997;14:492-7
5 Salisbury CJ, Tettersell MJ. Comparison of the work of a nurse practitioner with that of a general practitioner. $J R$ Coll Gen Pract 1988;38:314-6.

6 Beckman H, Kaplan SH, Frankel R. Outcome based research on doctorpatient communication: A review. In: Stewart M, Roter D, eds. Communicating with medical patients. Newbury Park: Sage Publications, 1989.

7 Kinnersley P. The patient-centredness of consultations and the relationship to outcomes in primary care [MD thesis]. Bristol: University of Bristol, 1997.

8 Baker R. Development of a questionnaire to assess patients' satisfaction with consultations in general practice. Br J Gen Pract 1990;40:487-90.

9 Poulton BC. Use of the consultation satisfaction questionnaire to examine patients' satisfaction with general practitioners and community nurses: reliability, replicability and discriminant validity. $\mathrm{Br} J$ Gen Pract 1996;46:26-31.

10 Clement MJ, Kinnersley P, Howard E, Turton P, Rogers C, Parry K, et al. A randomised controlled trial of nurse practitioner versus general practitioner care for patients with acute illnesses in primary care. Cardiff: Welsh Office of Research and Development, 1999.

11 Lewis CC, Scott DE, Pantell RH, Wolf MT. Parent satisfaction with children's medical care: development, field test and validation of a questionnaire. Med Care 1988;24:209-15.

12 Bland JM, Altman DG. Statistical methods for assessing agreement between two methods of clinical measurement. Lancet 1986; $: 307-10$.

13 Office of Population Censuses and Surveys. Standard occupational classification. Vols 1-3. London: HMSO, 1991

14 Royal College of General Practitioners. Morbidity statistics from general practice: third national study 1981-2. London: RCGP, Office of Population Censuses and Surveys, 1986

15 Kinnersley P, Stott N, Peters TJ, Harvey I. The patient-centredness of consultations and outcome in primary care. Br J Gen Pract 1999;49;711-6.

16 Helman CG. Culture, health and illness. Bristol: John Wright and Sons, 1984.

17 Howie JGR, Porter AMD, Heaney DJ, Hopton JL. Long to short consultation ratio: a proxy measure of quality of care for general practice. $\mathrm{BrJ} \mathrm{Gen}$ Pract 1991:41:48-54.

18 Stewart M. Studies of health outcomes and patient-centred communication. In: Stewart M, Brown JB, Weston WW, McWhinney IR, McWilliam CL, Freeman TR, eds. Patient-centred medicine-transforming the clinical method. Thousand Oaks, CA: Sage Publications, 1995.

(Accepted 17 February 2000)

\title{
Randomised controlled trial comparing cost effectiveness of general practitioners and nurse practitioners in primary care
}

\author{
P Venning, A Durie, M Roland, C Roberts, B Leese
}

University of

Manchester Schoo

of Primary Care,

Rusholme Health

Centre, Manchester

M14 5NP

P Venning

lecturer, practice

nursing

A Durie

research associate

National Primary

Care Research and

Development

Centre, University

of Manchester,

Manchester

M13 9PL

M Roland

professor of general

practice

B Leese

senior research fellow

continued over

BMJ 2000;320:1048-53

\author{
Abstract \\ Objective To compare the cost effectiveness of \\ general practitioners and nurse practitioners as first \\ point of contact in primary care. \\ Design Multicentre randomised controlled trial \\ of patients requesting an appointment the \\ same day. \\ Setting 20 general practices in England and Wales. \\ Participants 1716 patients were eligible for \\ randomisation, of whom 1316 agreed to \\ randomisation and 1303 subsequently attended the \\ clinic. Data were available for analysis on 1292 \\ patients (651 general practitioner consultations and \\ 641 nurse practitioner consultations). \\ Main outcome measures Consultation process \\ (length of consultation, examinations, prescriptions, \\ referrals), patient satisfaction, health status, return \\ clinic visits over two weeks, and costs. \\ Results Nurse practitioner consultations were \\ significantly longer than those of the general \\ practitioners (11.57 $7.28 \mathrm{~min}$; adjusted difference
}

$4.20,95 \%$ confidence interval 2.98 to 5.41 ), and nurses carried out more tests $(8.7 \%$ v $5.6 \%$ of patients; odds ratio $1.66,95 \%$ confidence interval 1.04 to 2.66) and asked patients to return more often $(37.2 \%$ v $24.8 \% ; 1.93,1.36$ to 2.73$)$. There was no significant difference in patterns of prescribing or health status outcome for the two groups. Patients were more satisfied with nurse practitioner consultations (mean score $4.40 v 4.24$ for general practitioners; adjusted difference $0.18,0.092$ to $0.257)$. This difference remained after consultation length was controlled for. There was no significant difference in health service costs (nurse practitioner $£ 18.11 v$ general practitioner $£ 20.70$; adjusted difference $22.33,-£ 1.62$ to $£ 6.28$ ).

Conclusions The clinical care and health service costs of nurse practitioners and general practitioners were similar. If nurse practitioners were able to maintain the benefits while reducing their return consultation rate or shortening consultation times, they could be more cost effective than general practitioners. 